\title{
Comparison of electrical nerve stimulation, electrical muscle stimulation and magnetic nerve stimulation to assess the neuromuscular function of the plantar flexor muscles
}

\author{
Daria Neyroud · John Temesi • Guillaume Y. Millet • \\ Samuel Verges $\cdot$ Nicola A. Maffiuletti $\cdot$ Bengt Kayser • \\ Nicolas Place
}

Received: 19 August 2014 / Accepted: 3 February 2015 / Published online: 15 February 2015

(C) Springer-Verlag Berlin Heidelberg 2015

\begin{abstract}
Introduction As it might lead to less discomfort, magnetic nerve stimulation (MNS) is increasingly used as an alternative to electrical stimulation methods. Yet, MNS and electrical nerve stimulation (ENS) and electrical muscle stimulation (EMS) have not been formally compared for the evaluation of plantar flexor neuromuscular function.

Methods We quantified plantar flexor neuromuscular function with ENS, EMS and MNS in 10 volunteers in fresh and fatigued muscles. Central alterations were assessed through changes in voluntary activation level (VAL) and peripheral function through changes in M-wave, twitch and doublet (PS100) amplitudes. Discomfort associated with $100-\mathrm{Hz}$ paired stimuli delivered with each method was evaluated on a $10-\mathrm{cm}$ visual analog scale.

Results VAL, agonist and antagonist M-wave amplitudes and PS100 were similar between the different methods in both fresh and fatigued states. Potentiated peak twitch
\end{abstract}

Communicated by Toshio Moritani.

D. Neyroud · B. Kayser $\cdot$ N. Place $(\square)$

Institute of Sport Sciences, Faculty of Biology and Medicine,

University of Lausanne, Quartier UNIL-Mouline, Building

Geopolis, Lausanne 1015, Switzerland

e-mail: Nicolas.place@unil.ch

D. Neyroud · B. Kayser · N. Place

Department of Physiology, Faculty of Biology and Medicine,

University of Lausanne, Lausanne, Switzerland

D. Neyroud · B. Kayser

Institute of Movement Sciences and Sports Medicine, University

of Geneva, Geneva, Switzerland

J. Temesi · G. Y. Millet

Université de Lyon, 42023 Saint-Etienne, France was lower in EMS compared to ENS, whereas no difference was found between ENS and MNS for any parameter. Discomfort associated with MNS $(1.5 \pm 1.4 \mathrm{~cm})$ was significantly less compared to ENS $(5.5 \pm 1.9 \mathrm{~cm})$ and EMS $(4.2 \pm 2.6 \mathrm{~cm})(p<0.05)$.

Conclusion When PS100 is used to evaluate neuromuscular properties, MNS, EMS and ENS can be used interchangeably for plantar flexor neuromuscular function assessment as they provide similar evaluation of central and peripheral factors in unfatigued and fatigued states. Importantly, electrical current spread to antagonist muscles was similar between the three methods while discomfort from MNS was much less compared to ENS and EMS. MNS may be potentially employed to assess neuromuscular function of plantar flexor muscles in fragile populations.

Keywords Muscle fatigue - Contractile properties . M wave $\cdot$ Voluntary activation $\cdot$ Discomfort

J. Temesi · G. Y. Millet

Human Performance Laboratory, Faculty of Kinesiology,

University of Calgary, Calgary, Canada

S. Verges

HP2 Laboratory, Grenoble Alpes University and INSERM

(U1042), Grenoble, France

N. A. Maffiuletti

Neuromuscular Research Laboratory, Schulthess Clinic,

Zurich, Switzerland 


$\begin{array}{ll}\text { Abbreviations } \\ \text { EMG } & \text { Electromyographic } \\ \text { EMS } & \text { Electrical muscle stimulation } \\ \text { ENS } & \text { Electrical nerve stimulation } \\ \text { GL } & \text { Gastrocnemius lateralis } \\ \text { GM } & \text { Gastrocnemius medialis } \\ \text { MNS } & \text { Magnetic nerve stimulation } \\ \text { MVC } & \text { Maximal voluntary contraction } \\ \text { PS10 } & \text { 10-Hz paired stimuli } \\ \text { PS100 } & \text { 100-Hz paired stimuli } \\ \text { Pt } & \text { Peak twitch } \\ \text { SD } & \text { Standard deviation } \\ \text { SE } & \text { Standard error } \\ \text { SOL } & \text { Soleus } \\ \text { TA } & \text { Tibialis anterior } \\ \text { VAL } & \text { Maximal voluntary activation level }\end{array}$

\section{Introduction}

Transcutaneous electrical stimulation is routinely used as a testing tool in both research and clinical settings to gain insight into neuromuscular function (Millet et al. 2012). The common techniques consist of electrically stimulating a muscle nerve trunk (electrical nerve stimulation, ENS) or directly depolarizing muscle terminal axonal branches through surface electrodes placed over the muscle (electrical muscle stimulation, EMS). Both these techniques present limitations restricting their wide application. The relatively high discomfort/pain often associated with ENS (Han et al. 2006; Place et al. 2010) limits its use in sensitive populations (children, elderly, patients). Additionally, concerns have been raised regarding the possible displacement of the stimulation electrode during voluntary contractions and the possible recruitment of antagonist muscles in certain muscle groups with ENS (Place et al. 2010). EMS partly overcomes these drawbacks but may lead to incomplete or different spatial recruitment in comparison to ENS (Martin et al. 2004; Place et al. 2010; Rodriguez-Falces et al. 2013a).

Magnetic nerve stimulation (MNS) is increasingly being used to assess neuromuscular function in muscles such as the knee extensors (Polkey et al. 1996; Swallow et al. 2007; Kremenic et al. 2009; Bachasson et al. 2013), adductor pollicis (Harris et al. 2000) and respiratory muscles (Man et al. 2004). Importantly, it has been found to be less painful than ENS and EMS for quadriceps muscle stimulation (Han et al. 2006). The reduced discomfort associated with MNS could represent a great advantage for testing sensitive populations. However, one of the main drawbacks of MNS arises from the limited maximal output of currently available magnetic stimulators. Supramaximal stimulation may thus not be achieved in all conditions (Tomazin et al. 2011) and might preclude the use of MNS to assess the neuromuscular function in fatigued conditions. As such, maximal stimulator output might be insufficient to recruit all motor units from the muscle group of interest, a prerequisite for neuromuscular evaluation in the fatigued state (Millet et al. 2011) when motoneuron excitation threshold is increased (Kernell and Monster 1982a, b; Vagg et al. 1998; Burke 2002). This limitation may also be a technical problem in overweight individuals if a thick layer of subcutaneous fat tissue is present at the stimulation site (Tomazin et al. 2011). Furthermore, since the magnetically-induced electric field has been shown to decrease less with tissue depth compared to electrical stimulation (Barker 1991), MNS might result in increased antagonist muscle recruitment possibly leading to underestimation of the evoked force.

Despite the growing use of MNS, only a few studies have compared it to ENS (Harris et al. 2000; Laghi et al. 1996; Verges et al. 2009) or EMS (Han et al. 2006; Verges et al. 2009) and even fewer have examined the validity of MNS in the fatigued state (Verges et al. 2009). Similar results have been reported for resting peak twitch assessment (Harris et al. 2000) and peripheral fatigue detection between MNS and ENS (Laghi et al. 1996), whereas less perceived discomfort was found with MNS compared to EMS (Han et al. 2006). Conversely, the comparison between EMS and ENS for neuromuscular function assessment has been extensively studied (Scaglioni and Martin 2009; Martin et al. 2004; Rutherford et al. 1986; Place et al. 2010; Rodriguez-Falces et al. 2013a, b). Most of these studies were conducted on the knee extensors and reported similar outcomes between ENS and EMS for both mechanical and electromyographic activity responses (Rodriguez-Falces et al. 2013a, b; Rutherford et al. 1986; Martin et al. 2004; Place et al. 2010). Scaglioni and Martin (2009) observed similar maximal voluntary activation levels (VAL) and evoked forces with ENS and EMS in the plantar flexors. Only one study compared all three stimulation techniques (ENS, EMS and MNS) in the knee extensors of healthy individuals and found similar neuromuscular properties in fresh and fatigued muscle states (Verges et al. 2009). However, no studies have investigated the validity of MNS and EMS compared to ENS in the plantar flexors, a functionally important group of postural muscles. Impairment of plantar flexor function greatly impairs quality of life in many disorders such as cerebral palsy (Brouwer et al. 1998; Wiley and Damiano 1998), Charcot-MarieTooth disease (Vinci and Perelli 2002), Duchenne muscular dystrophy (Gaudreault et al. 2009) and diabetic polyneuropathy (Andreassen et al. 2009). If validated, MNS would be a useful means to assess neuromuscular function in such patients.

The main purpose of this study was to determine whether the evaluation of plantar flexor neuromuscular function with MNS, EMS and ENS provides similar results in fresh and fatigued states in healthy and physically active 
individuals. The two following hypotheses were tested: (1) MNS, EMS and ENS lead to similar estimates of central and peripheral determinants of neuromuscular function in both fresh and fatigued states and (2) MNS causes less discomfort compared to EMS/ENS.

\section{Materials and methods}

\section{Subjects}

Ten healthy and physically active subjects ( 7 men-3 women, $35 \pm 10$ years, $173 \pm 6 \mathrm{~cm}, 71 \pm 10 \mathrm{~kg}$ ) volunteered to participate in this study after having been informed of the experimental procedures and possible risks. All subjects were recreationally physically active but not involved in any structured training program. Additionally, all participants had previously taken part in at least one similar experiment using comparable testing procedures. The study protocol was performed in accordance with the Declaration of Helsinki and approved by the Geneva University Hospital ethics committee (protocol 11-287). Before participation, each subject gave written informed consent.

\section{Experimental protocol}

Subjects lay prone with their dominant leg (as assessed via the revised Waterloo questionnaire (Elias et al. 1998)) fully extended so that the popliteal fossa was accessible for MNS. The dominant foot was securely strapped at the level of the ankle and metatarsi to a pedal fixed to a wall and equipped with a strain gauge (S2 1,000 N, sensitivity $2 \mathrm{mV} / \mathrm{V}$ and $0.0043 \mathrm{~V} / \mathrm{N}$; HBM, Darmstadt, Germany). Ankle angle was set at $90^{\circ}$. To limit upper body contribution to the force production, straps were applied at the hip level. The force signal was recorded at $1 \mathrm{kHz}$ using an analog-digital conversion system (MP150, BIOPAC, Goleta, USA). The experimental procedure started by determining the maximal stimulus intensity for ENS and EMS, i.e. the intensity where a plateau in peak twitch evoked force and agonist M-wave amplitudes was reached in relaxed conditions (see electromyographic (EMG) recordings for further details). Recruitment curves relating force to stimulation intensity were obtained by evoking single stimuli of increasing (or decreasing) intensity delivered between 50 and $120 \%$ of maximal intensity (steps $5 \%$ of maximal intensity) for ENS and EMS, and from 50 to $100 \%$ of maximal stimulator output for MNS (Verges et al. 2009). The increasing or decreasing pattern and the order in which ENS, MNS and EMS were delivered were counterbalanced between subjects and kept constant for a given subject throughout the protocol. All subsequent stimuli were delivered at $120 \%$ of the maximal intensity for ENS and EMS and at $100 \%$ of maximal stimulator output for MNS. Subjects then warmed-up by contracting their plantar flexors $8-10$ times at $20-80 \%$ of their estimated maximal voluntary contraction (MVC) force. The pre-exercise tests consisted of 3 MVCs (3-4 s) with $100-\mathrm{Hz}$ paired stimuli delivered at maximal force by EMS, ENS and MNS (1 MVC for each stimulation method, see Fig. 1). Two seconds after each MVC, 100- and 10-Hz paired stimuli were delivered at an interval of $2 \mathrm{~s}$ in the relaxed muscle with the stimulation technique used during the MVC. Subjects then performed a fourth MVC that was followed by single stimuli delivered sequentially by EMS, ENS and MNS with an inter-stimulus interval of $2 \mathrm{~s}$. Thereafter, a fatiguing task, consisting of four 40-s MVCs of the plantar flexors separated by $8 \mathrm{~s}$, was started. During the 8 -s rest between MVCs, three twitches were evoked by the three stimulation methods with an inter-stimulus interval of $2 \mathrm{~s}$. Immediately after the fatiguing task, post-exercise tests (which were identical to the pre-exercise tests) were completed. An outline of the experimental protocol is provided in Fig. 1.

\section{Electrical nerve stimulation}

ENS was delivered transcutaneously by a cathode self-adhesive electrode (1-cm diameter, with no applied pressure, Meditrace 100, Tyco, Markham, Canada) placed over the tibial nerve in the popliteal fossa and an anode $(5 \times 10 \mathrm{~cm}$, Compex, Ecublens, Switzerland) located over the anterior surface of the knee, $2-3 \mathrm{~cm}$ proximal to the patella. Rectangular-wave pulses (1-ms duration) were generated by a high-voltage (max $400 \mathrm{~V})$ constant-current stimulator (Digitimer DS7AH, Hertfordshire, UK) as single and paired electrical stimuli. Maximal intensity (i.e. the stimulus intensity at which no further increase in peak twitch or M-wave amplitudes was observed despite an increase of two 10-mA increments) was determined (Neyroud et al. 2014). All stimuli were subsequently delivered at $120 \%$ of maximal intensity (except during the recruitment curve) to ensure supramaximality ( $157 \pm 37 \mathrm{~mA}$, range 120-228 mA).

\section{Electrical muscle stimulation}

EMS was delivered transcutaneously to the plantar flexors via two $5 \times 10 \mathrm{~cm}$ rectangular self-adhesive surface electrodes (Compex). The anode was placed over the gastrocnemii ( $\sim 5 \mathrm{~cm}$ distal to the popliteal fossa) and the cathode over the soleus ( $\sim 10 \mathrm{~cm}$ proximal to the calcaneus). Pulse width was $1 \mathrm{~ms}$ as for ENS. Both single and paired stimuli were delivered by the same stimulator as for ENS. Stimulus intensity was set at $120 \%$ of maximal intensity $(181 \pm 24 \mathrm{~mA}$, range 144-216 mA), except during the recruitment curve. 
Fig. 1 Experimental protocol. The order in which the different methods of stimulation were delivered was kept constant for a given subject and counterbalanced between subjects. In this example, black arrows depict electrical nerve stimulation, grey arrows depict electrical muscle stimulation and dark diamond arrows depict magnetic nerve stimulation. ENS electrical nerve stimulation, EMS electrical muscle stimulation, MNS magnetic nerve stimulation

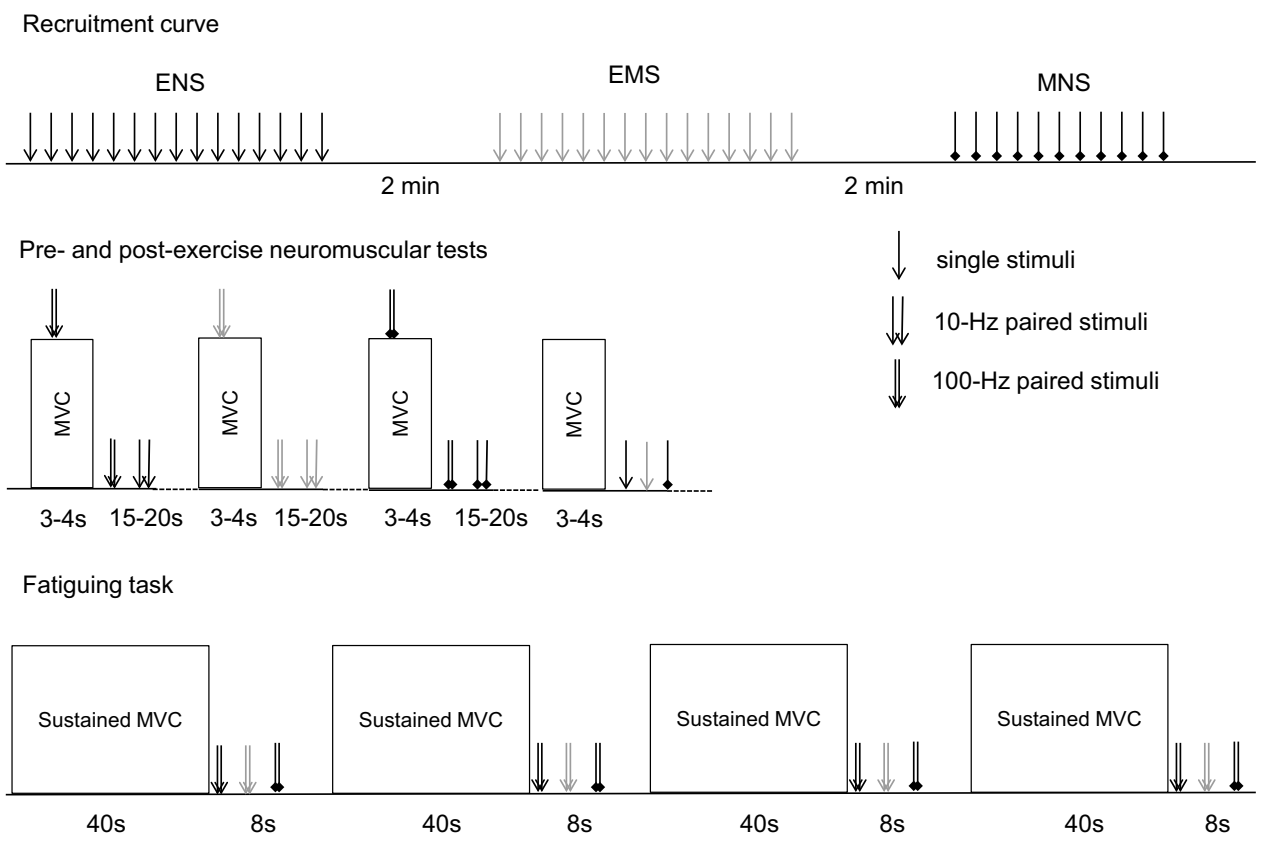

Magnetic nerve stimulation

MNS was delivered to the tibial nerve with a 45-mm figureof-eight coil (peak magnetic field strength $2.5 \mathrm{~T}$ ) positioned over the popliteal fossa and powered by two Magstim $200^{2}$ stimulators connected by the Bistim ${ }^{2}$ module (Magstim, Whitland, Dyfed, UK). The stimulus duration was $1 \mathrm{~ms}$ with a rise time of $100 \mu \mathrm{s}$. The coil position where the largest peak twitch and M-waves were elicited was marked on the skin. All stimuli were delivered at the maximal stimulator output (Verges et al. 2009), except during the recruitment curve.

\section{Electromyographic recordings}

EMG activity was recorded from the agonist soleus (SOL), gastrocnemius lateralis (GL), gastrocnemius medialis (GM) and antagonist tibialis anterior (TA) muscles with pairs of circular silver chloride $(\mathrm{Ag} / \mathrm{AgCl})$ (1-cm recording diameter) self-adhesive electrodes (Meditrace 100) with an inter-electrode (center-to-center) distance of $3 \mathrm{~cm}$. For the SOL, the electrodes were placed distally at a location corresponding to $2 / 3$ of the distance between the medial condyle of the femur and the medial malleolus. For GL, GM and TA, the electrodes were positioned lengthwise over the middle of the muscle belly (Place et al. 2009). The reference electrode was placed over the ipsilateral patella.

Low resistance between pairs of electrodes was obtained by shaving, lightly abrading and cleaning the skin. EMG signals were amplified with a gain of 1,000 , filtered with a bandwidth frequency between 10 and $500 \mathrm{~Hz}$, digitized at a sampling frequency of $2 \mathrm{kHz}$ and recorded with the analog-digital conversion system. Isometric force and EMG data were stored and analyzed offline with commercially available software (Acqknowledge, BIOPAC Systems, Goleta, USA).

\section{Discomfort}

During pre-exercise neuromuscular assessment, a horizontal visual analog scale $(10 \mathrm{~cm})$ was presented to the subjects after each MVC to assess the discomfort associated with $100-\mathrm{Hz}$ paired stimuli delivered in a relaxed state. Subjects were asked to place a vertical mark between "no discomfort" (0 cm) and "worst possible discomfort" $(10 \mathrm{~cm})$ to rate the discomfort associated with the stimulation.

Data analysis

\section{Force data}

Maximal isometric MVC force was considered as the peak force attained during the MVC. Muscle fatigue was defined as the amount of MVC force loss induced after exercise (Gandevia 2001). As an index of muscle activation, VAL during MVCs was quantified as follows:

$\mathrm{VAL}=(1-($ superimposed $100-\mathrm{Hz}$ doublet amplitude/ resting potentiated $100-\mathrm{Hz}$ doublet amplitude) $) \times 100$ (Allen et al. 1995).

A correction was consistently applied to this equation when the superimposed doublet was not elicited at peak force (Strojnik and Komi 1998). Pre- (unpotentiated and 
potentiated) and post-exercise twitch (Pt) and 10 and 100$\mathrm{Hz}$ paired stimuli (PS10 and PS100, respectively) amplitudes were analyzed to assess muscle contractile properties. The PS10/100 ratio was calculated both pre- and post-exercise for each method of stimulation and the change in this ratio was used as an index of low-frequency fatigue (Verges et al. 2009).

\section{EMG data}

Due to stimulation artifact contamination, GL and GM M waves could be consistently recorded with the three methods of stimulation in only 4 and 6 individuals, respectively, and were thus not considered for the analyses. Whenever possible, all agonist M-wave amplitudes were considered for the determination of stimulation intensity. When GL and/or GM M waves could not be obtained, only uncontaminated $\mathrm{M}$ waves were considered for stimulation intensity determination. SOL M-wave peak-to-peak amplitude was measured from the EMG responses elicited by single stimuli before and after the fatiguing task. Antagonist M-wave amplitude elicited by the agonist nerve/muscle stimulation was measured to assess the level of current spread to TA innervation.

\section{Statistical analysis}

Data normality was checked with the Shapiro-Wilk test. Depending on the normality test outcome, oneway repeated measures ANOVAs (intensity of stimulation) or Friedman tests (when normality was not present, i.e. for EMS and MNS Pt and for ENS and EMS SOL M-wave amplitudes) were performed to compare Pt and SOL M-wave amplitudes obtained with each of the three methods of stimulation (between 50 and $120 \%$ of maximal intensity for ENS and EMS, and between 50 and $100 \%$ of maximal stimulator output for MNS), as well as for all pre-exercise parameters and for relative changes in those parameters induced by the fatiguing task. One-way repeated measures ANOVAs (method of stimulation) were also conducted to compare $\mathrm{Pt}$ and $\mathrm{M}$-wave amplitudes at maximal stimulation intensity/stimulator output. To evaluate the extent of fatigue measured by each method of stimulation, two-way [method of stimulation (ENS vs. EMS vs. MNS) $\times$ time (pre- vs. post-exercise)] repeated measures ANOVAs were performed for all parameters. For all ANOVAs, when significant main effects were found, the Tukey post-hoc test was used to identify differences among pairs of means. The $\alpha$-level for statistical significance was set at $p<0.05$. Bland-Altman analyses were also conducted to assess the agreement between the different methods of stimulation for the principal parameters (i.e. VAL, PS100, $\mathrm{Pt}$ and SOL M-wave amplitude). Sigmaplot software for
Windows (version 11, Systat, Chicago, IL) was used for statistical analysis. Data are reported as mean \pm SD in text and tables and as mean $\pm \mathrm{SE}$ in figures.

\section{Results}

\section{Recruitment curves}

Similar to ENS and EMS, a plateau in unpotentiated Pt could successfully be reached in MNS, as Pt forces were similar between 70 and $100 \%$ of maximal stimulator output for MNS ( $p>0.05$, Fig. 2a). Furthermore, MNS Pt recorded at $100 \%$ of maximal stimulator output $(118.6 \pm 25.1 \mathrm{~N})$ was similar to ENS $(116.2 \pm 26.3 \mathrm{~N})$ and EMS $(109.4 \pm 31.8 \mathrm{~N}) \mathrm{Pt}$ obtained at $100 \%$ of maximal intensity $(p>0.05)$. Additionally, similar to ENS and EMS, SOL M-wave amplitudes reached a plateau with MNS as no differences were observed between 80 and $100 \%$ of maximal stimulator output ( $p>0.05$, Fig. $2 b)$. No differences were found in TA M-wave amplitudes at maximal stimulation intensity/stimulator output between ENS, EMS and MNS ( $p>0.05$, Fig. 2c).

\section{Discomfort}

Discomfort induced by the paired-pulse stimuli was found to be significantly $(p<0.001)$ lower for MNS $(1.5 \pm 1.4 \mathrm{~cm})$ compared to ENS $(5.5 \pm 1.9 \mathrm{~cm})$ and EMS $(4.2 \pm 2.6 \mathrm{~cm}$, Fig. 3), with no difference between ENS and EMS $(p>0.05)$.

\section{Neuromuscular function evaluation}

Typical recordings of the plantar flexor voluntary and evoked forces and SOL M waves elicited by single stimuli with the three methods of stimulation are depicted in Fig. 4.

With each method of stimulation, VAL, M-wave and contractile properties were assessed in comparable conditions at a given time point (in fresh or fatigued states), i.e. at similar MVC force levels for the three stimulation techniques, in those states, respectively $(p>0.05)$.

Prior to exercise, a main effect of stimulation method was only found for Pt (potentiated by the MVC), which was lower in EMS compared to ENS (Table 1) and for PS10 (the post hoc test could not identify any differences between the different methods of stimulation). The BlandAltman analysis also showed that the different methods of stimulation allowed a similar assessment of VAL, PS100, $\mathrm{Pt}$ and SOL M-wave amplitudes despite a slight systematic bias with MNS for VAL assessment (Fig. 5).

After the completion of the fatiguing task, a significant MVC force decrease of $9.8 \pm 15.9 \%(p<0.05)$ 

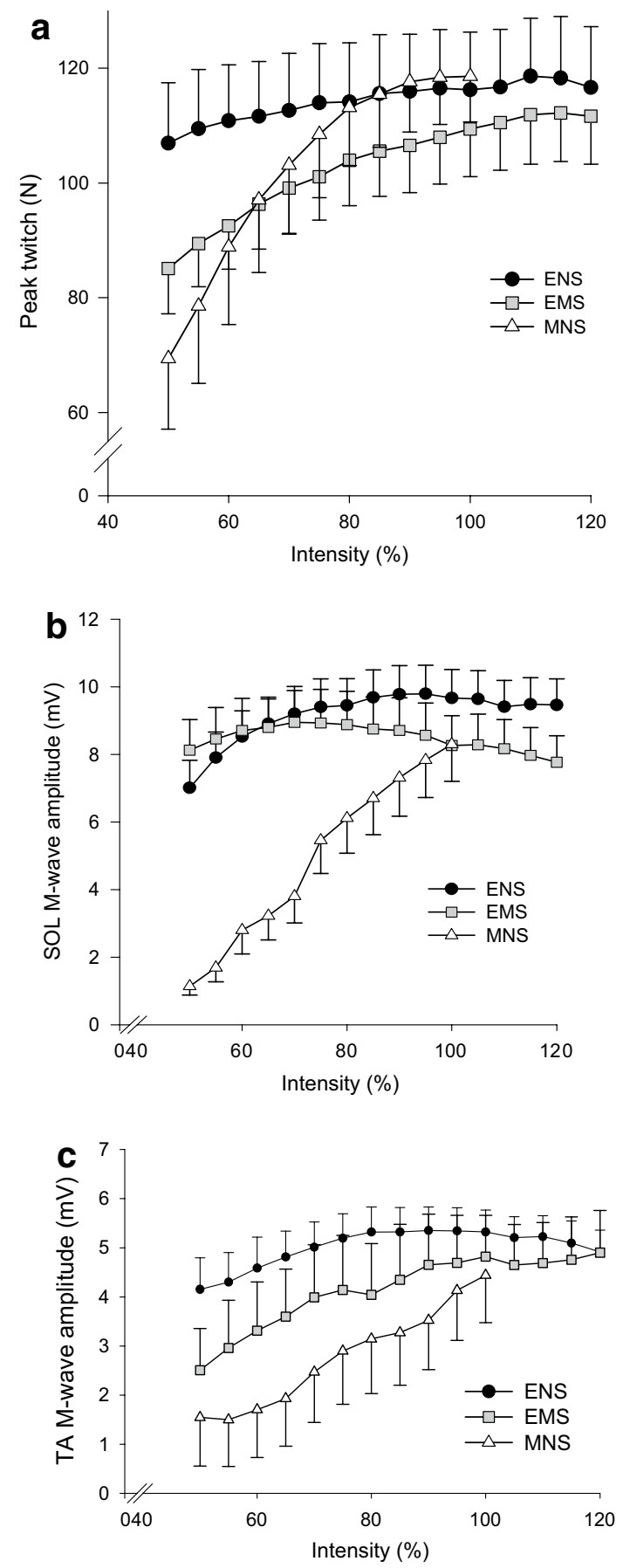

Fig. 2 a Peak twitch, b soleus M-wave amplitudes and c tibialis anterior M-wave amplitudes evoked via ENS, MNS and EMS for intensities ranging from 50 to $120 \%$ of maximal intensity in ENS and EMS and from 50 to $100 \%$ of maximal stimulator output for MNS. Note that for clarity purposes significant differences at submaximal intensities are not indicated. EMS and MNS Pt as well as ENS and EMS SOL M-wave amplitudes were not normally distributed. For TA, $N=7$ as $\mathrm{M}$ waves were contaminated by stimulation artifacts in 3 subjects. ENS electrical nerve stimulation, EMS electrical muscle stimulation, $M N S$ magnetic nerve stimulation

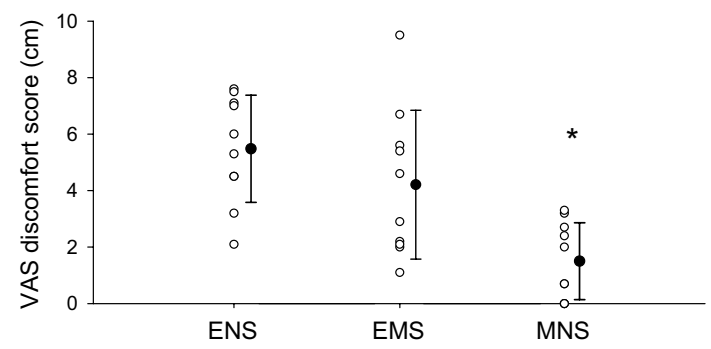

Fig. 3 Discomfort associated with paired $100-\mathrm{Hz}$ stimuli delivered by ENS, EMS and MNS. Empty circles represent individual data and black filled circles represent mean data \pm SE. ${ }^{*} p<0.05$ indicates significantly lower discomfort for MNS compared to ENS and EMS. ENS electrical nerve stimulation, EMS electrical muscle stimulation, MNS magnetic nerve stimulation

was observed. At the central level, no VAL changes were found $(p>0.05)$. At the peripheral level, SOL and TA M-wave amplitudes as well as potentiated Pt, PS100 and PS10 amplitudes were unchanged by exercise $(p>0.05)$, whereas a significant (but similar between methods) reduction in PS10/100 ratio was observed ( $p<0.05$, Fig. 6). This exercise-induced fatigue was not differently detected by the three stimulation methods ( $p>0.05$, Table 2). Bland-Altman analysis also showed a good agreement between the different methods of stimulation in the post-exercise condition (Fig. 5).

During the fatiguing task, the potentiated $\mathrm{Pt}$ and the associated SOL M-wave evoked after each 40-s sustained MVC remained unchanged and were comparable across all stimulation methods (data not shown).

\section{Discussion}

The present study was designed to assess whether MNS, EMS and ENS can be used interchangeably to evaluate neuromuscular function of fresh and fatigued plantar flexors in healthy individuals. Whereas ENS and MNS led to a similar evaluation of the neuromuscular function, EMS resulted in an underestimation of potentiated Pt in comparison to ENS in the unfatigued state. Nevertheless, when the $100-\mathrm{Hz}$ paired stimuli were considered, EMS was found to provide a comparable evaluation of neuromuscular function to ENS and MNS in both fresh and fatigued states. Further, in accordance with our hypothesis, MNS induced much less discomfort compared to ENS, whereas the discomfort associated with EMS and ENS was similar. 
Fig. 4 Typical recordings of a MVC and superimposed and resting doublets and $\mathbf{b}$ potentiated twitches evoked by each stimulation method and c the associated SOL M wave. Dashed lines indicate the windows in which $\mathrm{M}$ waves were searched
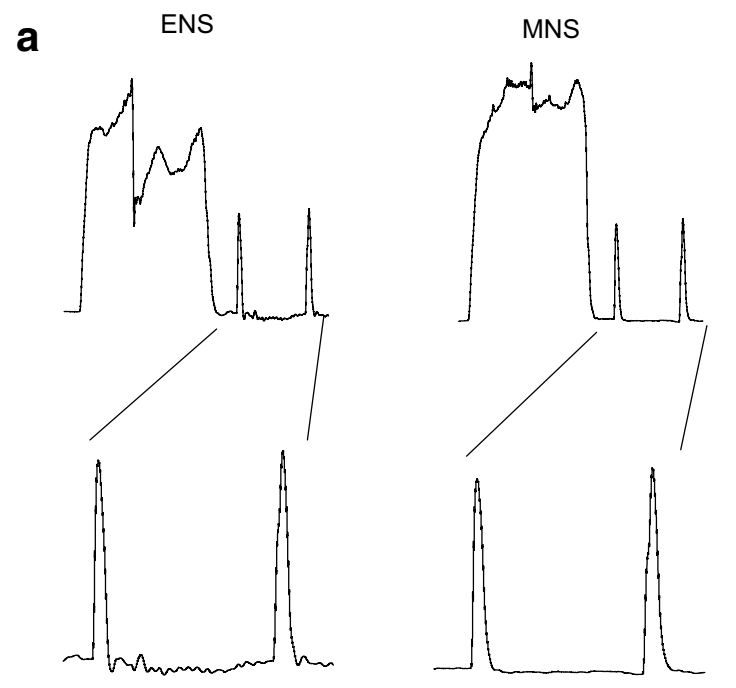

b
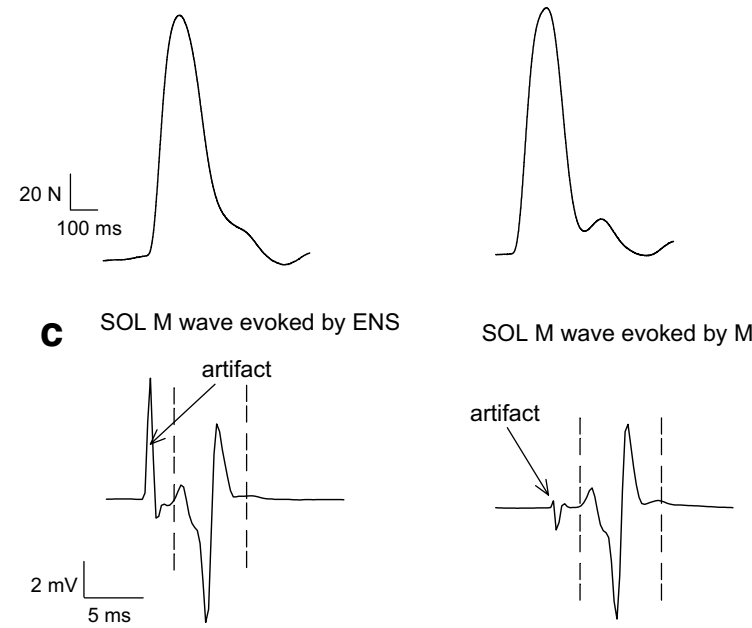

EMS

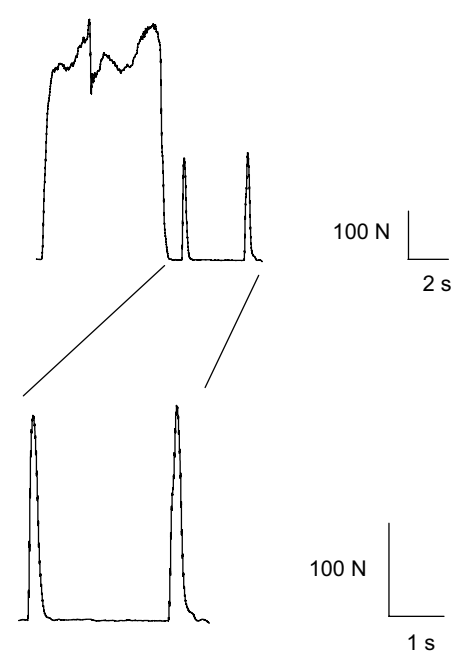

Peak twitch evoked by EMS

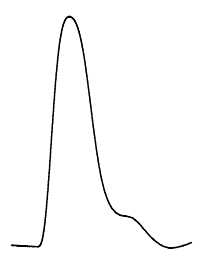

SOL M wave evoked by MNS
SOL M wave evoked by EMS

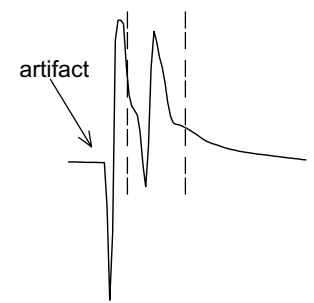

Table 1 Neuromuscular function assessment with ENS, EMS and MNS

\begin{tabular}{|c|c|c|c|c|}
\hline & ENS & EMS & MNS & Main effect \\
\hline $\operatorname{MVC}(\mathrm{N})$ & $621.5 \pm 169.3$ & $604.1 \pm 190.5$ & $600.0 \pm 171.3$ & 0.459 \\
\hline \multicolumn{5}{|l|}{ Central } \\
\hline VAL $(\%)$ & $75.9 \pm 19.1$ & $77.1 \pm 18.7$ & $68.0 \pm 20.3$ & 0.068 \\
\hline \multicolumn{5}{|l|}{ Peripheral } \\
\hline SOL M-wave amplitude (mV) & $10.0 \pm 3.5$ & $8.2 \pm 2.5$ & $7.7 \pm 3.9$ & 0.114 \\
\hline TA M-wave amplitude (mV) & $5.0 \pm 1.9$ & $4.7 \pm 2.8$ & $3.2 \pm 2.8$ & 0.087 \\
\hline $\operatorname{Pt}(\mathrm{N})$ & $168.6 \pm 25.1$ & $153.8 \pm 18.0^{*}$ & $159.2 \pm 25.9$ & 0.043 \\
\hline PS100 (N) & $237.1 \pm 36.0$ & $222.6 \pm 29.5$ & $218.2 \pm 57.6$ & 0.506 \\
\hline PS10 (N) & $262.4 \pm 36.4$ & $228.4 \pm 42.1$ & $229.8 \pm 49.7$ & 0.042 \\
\hline
\end{tabular}

For PS10 evoked by EMS, $N=9$ as no PS10 was evoked in one subject due to technical problems

ENS electrical nerve stimulation, EMS electrical muscle stimulation, $M N S$ magnetic nerve stimulation, $V A L$ voluntary activation level, $S O L$ soleus, TA tibialis anterior, Pt peak twitch, PS100 100-Hz paired-stimuli evoked force, PS10 10-Hz paired stimuli evoked force

$* p<0.05$ indicates a significant difference compared to ENS. Note that a main effect for stimulation method was found for PS10, but post hoc analysis did not reveal any difference between the means 

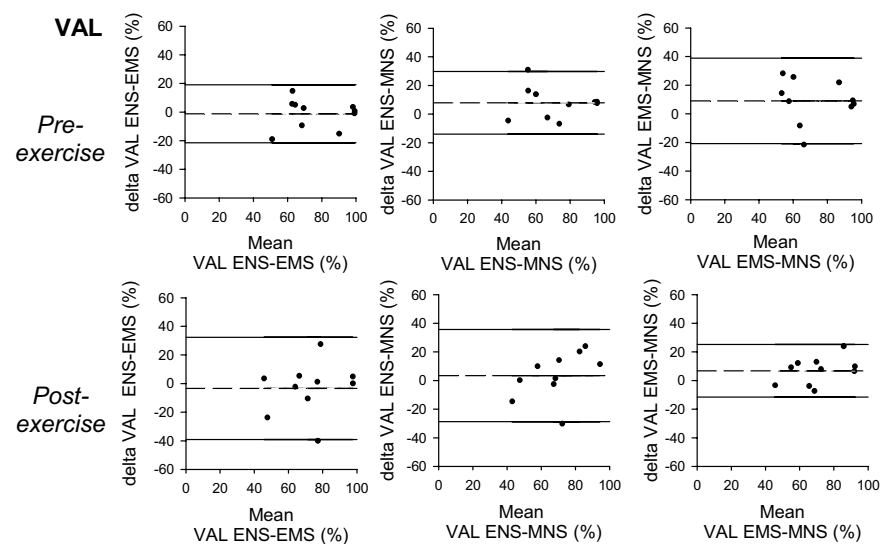

PS100
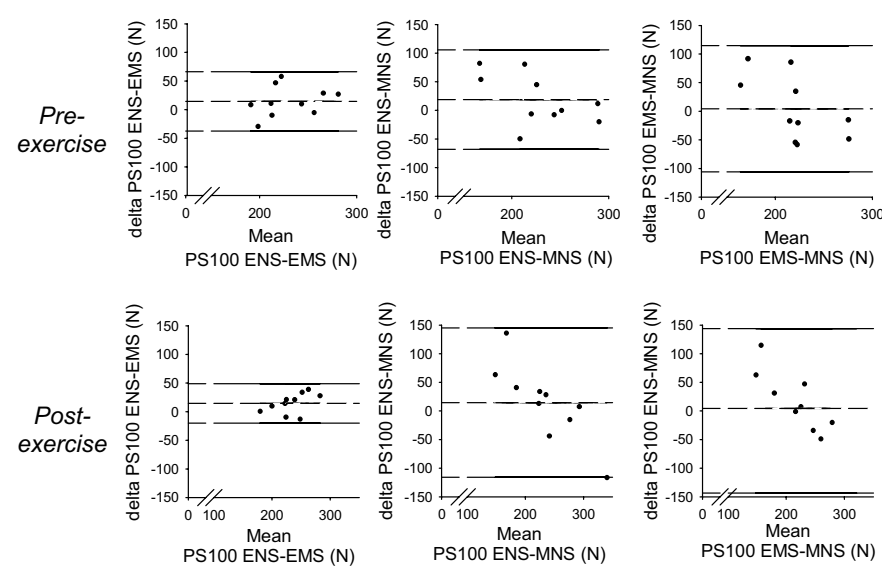
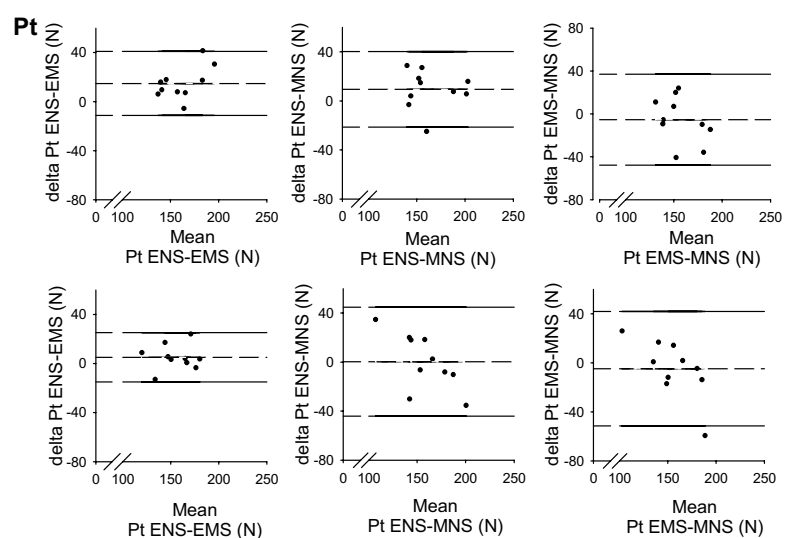

SOL
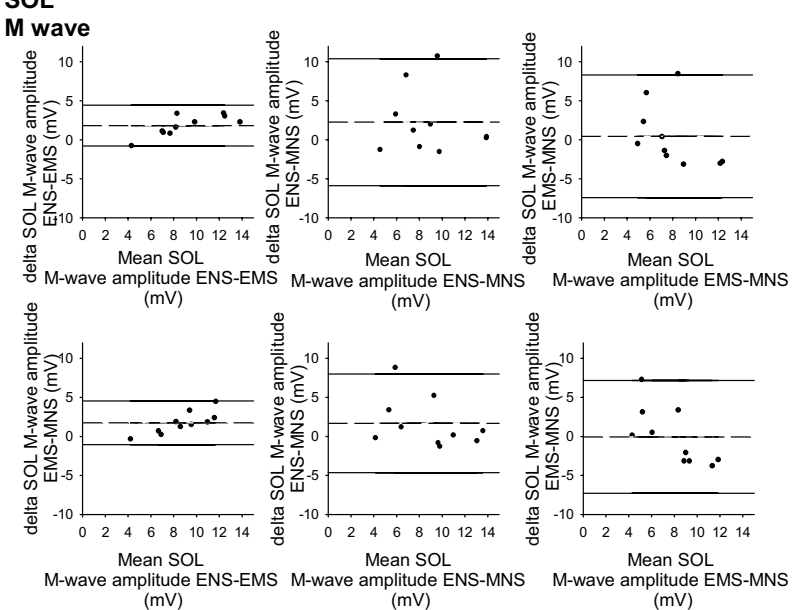

Fig. 5 Bland-Altman plots for voluntary activation level (VAL), $100-\mathrm{Hz}$ paired stimuli (PS100), peak twitch (Pt) and SOL M-wave amplitudes. Dashed lines represent the mean difference between the two considered techniques and solid lines represent the upper and lower limit of agreement. ENS electrical nerve stimulation, EMS electrical muscle stimulation, $M N S$ magnetic nerve stimulation

\section{Recruitment curves}

One of the major concerns related to the use of MNS and EMS compared to ENS for neuromuscular function assessment is the potentially incomplete motor unit recruitment with maximal stimulation intensity (Martin et al. 2004; Rodriguez-Falces et al. 2013a; Place et al. 2010). When stimulating with MNS, the capacity of the commercially available stimulators may be insufficient to elicit a maximal response (Tomazin et al. 2011; Millet et al. 2012), whereas the potentially incomplete motor unit recruitment with EMS could be due to the stimulation of superficial axonal terminal branches only (Rodriguez-Falces et al. 2013a). To address this issue, recruitment curves were plotted for the three methods of stimulation to verify the presence of a plateau for both Pt and SOL M-wave amplitudes. Additionally, $\mathrm{Pt}$ and SOL M-wave amplitudes obtained at $120 \%$ of maximal intensity for ENS and EMS and at $100 \%$ of maximal stimulator output for MNS were compared. A plateau was observed with all three stimulation methods. Further, unpotentiated $\mathrm{Pt}$ and SOL M-wave amplitudes associated with the maximal intensity of stimulation/stimulator output for the three methods of stimulation were similar. Importantly, if maximality is defined as no more than a $5 \%$ increase in Pt amplitudes between 90 and $100 \%$ of maximal intensity/stimulator output, this was successfully achieved in all subjects. Taken together, these results highlight complete and similar motor unit recruitment with ENS, EMS and MNS, which is in accordance with previous studies on knee extensors in healthy individuals where complete motor unit recruitment with ENS (Place et al. 2010; Verges et al. 2009; Rodriguez-Falces et al. 2013a) and MNS (Polkey et al. 1996; Verges et al. 2009; Tomazin et al. 2011) was also observed. Similarly, the comparable extent of plantar flexor motor unit recruitment between ENS and EMS is in agreement with results found in the knee extensors (Rodriguez-Falces et al. 2013a) but contrasts with other studies also performed on the knee extensors (Verges et al. 2009; Place et al. 2010). The discrepancies between the study of Rodriguez-Falces et al. 


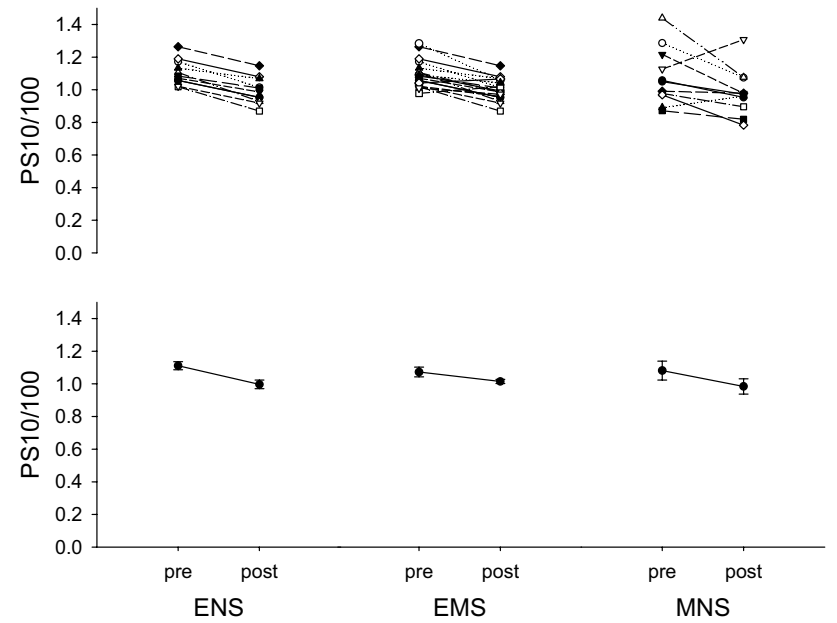

Fig. 6 PS10/100 ratio in pre- and post-exercise. Individual data are presented in the upper panel and group data in the lower panel. Postexercise PS10/100 ratio was significantly lower than pre-exercise with a significant main effect for time $(p<0.05)$. ENS electrical nerve stimulation, EMS electrical muscle stimulation, $M N S$ magnetic nerve stimulation, pre pre-exercise and post post-exercise. $N=10$ for ENS and MNS, and 9 for EMS as no PS10 was evoked pre-exercise in one subject

(2013a) and the ones of Verges et al. (2009) and Place et al. (2010) might be explained by different stimulation electrode sizes. Rodriguez-Falces et al. (2013a) used larger electrodes resulting in a greater stimulation area. This suggests that whereas EMS may not completely recruit large muscle groups such as the knee extensors, it may consistently recruit all motor units in smaller muscle groups such as the plantar flexors in healthy, physically active subjects.

Additionally, the present data did not reveal any differences between ENS and EMS at $120 \%$ of the maximal stimulation intensity and MNS at $100 \%$ of the magnetic stimulator output in current spread to the antagonist muscle, as highlighted by similar TA M-wave amplitudes. It thus appears that the greater size of the stimulating coil in MNS compared to the electrodes used for ENS and EMS is not problematic for antagonist current spread and thus presumably did not result in an underestimation of evoked plantar flexor forces (Place et al. 2010). Overall, MNS, EMS and ENS thus resulted in complete motor unit recruitment in healthy physically active individuals and similar current spread to the antagonist muscle.

\section{Neuromuscular function assessment}

In both fresh and fatigued conditions, ENS, EMS and MNS yielded similar results for PS100, PS10/100, SOL M-wave amplitudes and VAL. Potentiated Pt and PS10 were the only parameters showing a stimulation method main effect in the fresh state. While the post-hoc analysis could not identify a difference between the three methods of stimulation for PS10, Pt was found to be smaller when evoked by EMS compared to ENS. Further studies are required to uncover the reason for this underestimation of Pt when evoked by EMS as it cannot be explained in the present work. Nevertheless, for PS100-which is generally considered a better index of peripheral fatigue (Place et al. 2007)—no differences were found between ENS, EMS and MNS. The reason for this discrepancy between Pt and PS100 is unclear. Yet, it must be noted that a systematic bias was found with MNS for VAL assessment (as shown by the Bland-Altman analysis) and thus caution must be taken when interpreting VAL assessed via MNS. Thus, it appears that MNS might be used instead of ENS if contractile properties are assessed with PS100 (and not with $\mathrm{Pt}$ ) in both fresh and fatigued conditions as long as one keeps in mind a possible slight underestimation of VAL. The similar results found for ENS and MNS for all neuromuscular parameters at rest and their changes with

Table 2 Central and peripheral changes induced by the fatiguing task assessed with ENS, EMS and MNS

\begin{tabular}{|c|c|c|c|c|}
\hline & ENS & EMS & MNS & Main effect \\
\hline$\triangle \mathrm{MVC}$ force $(\%)$ & $-10.6 \pm 17.9$ & $-10.1 \pm 17.7$ & $-9.0 \pm 14.4$ & 0.784 \\
\hline \multicolumn{5}{|l|}{ Central } \\
\hline$\triangle \operatorname{VAL}(\%)$ & $-5.9 \pm 23.0$ & $-2.3 \pm 17.8$ & $2.9 \pm 23.0$ & 0.855 \\
\hline \multicolumn{5}{|l|}{ Peripheral } \\
\hline$\Delta$ SOL M-wave amplitude (\%) & $-1.9 \pm 12.3$ & $-1.5 \pm 13.7$ & $1.2 \pm 29.7$ & 0.741 \\
\hline$\Delta$ TA M-wave amplitude (\%) & $6.3 \pm 19.1$ & $-8.6 \pm 25.2$ & $-7.8 \pm 31.5$ & 0.569 \\
\hline$\Delta \mathrm{Pt}(\%)$ & $-5.4 \pm 13.2$ & $-0.2 \pm 11.8$ & $-1.3 \pm 14.8$ & 0.199 \\
\hline$\Delta \mathrm{PS} 100(\%)$ & $2.0 \pm 10.4$ & $2.6 \pm 13.7$ & $6.3 \pm 25.2$ & 0.987 \\
\hline$\Delta \mathrm{PS} 10(\%)$ & $-8.5 \pm 9.5$ & $4.6 \pm 30.8$ & $-7.5 \pm 19.2$ & 0.104 \\
\hline
\end{tabular}

For PS10 evoked by EMS, $N=9$ as no PS10 was evoked pre-exercise in one subject

ENS electrical nerve stimulation, EMS electrical muscle stimulation, $M N S$ magnetic nerve stimulation, VAL voluntary activation level, SOL soleus, TA tibialis anterior, Pt peak twitch, PS100 100-Hz paired-stimuli evoked force and PS10 10-Hz paired stimuli evoked force 
fatigue are in agreement with previous findings obtained for the knee extensors (Verges et al. 2009). Other studies have also shown the validity of MNS for neuromuscular function evaluation in the fatigued state for the knee extensors (Polkey et al. 1996) and the diaphragm (Laghi et al. 1996; Polkey et al. 2000). Thus, MNS appears to represent a valid method to assess neuromuscular adjustments of the plantar flexors. Regarding EMS, the present results are in agreement with previous studies showing similar assessment of VAL (Scaglioni and Martin 2009; Rutherford et al. 1986; Martin et al. 2004) and low-frequency fatigue (Martin et al. 2004) compared to ENS. Noteworthy, and despite the general good agreement observed between the different methods of stimulation, MNS appears more variable than ENS and EMS, as can be observed by the larger upper and lower limits of agreement.

The major difference observed between the three methods of stimulation was much less discomfort with MNS compared to ENS and EMS. This result is consistent with Han et al. (2006), who showed that for a similar evoked force, MNS resulted in less discomfort. This greater tolerance for MNS has been suggested to result from the fact that when MNS is used, the electric field is produced only once the magnetic field has passed the cutaneous nociceptors (Barker et al. 1987; Barker 1991). Indeed, the magnetic field produced by the stimulator passes through the different tissues with little attenuation, contrary to the electric field produced by ENS and EMS (Barker et al. 1987; Barker 1991). This allows stimulating deeper structures without the need of ENS and EMS for a high surface electric field to compensate for progressive intensity loss while penetrating the tissue. It is noteworthy that no difference was observed between VAS associated with ENS or EMS in contrast to the findings of Martin et al. (2004). This discrepancy can be explained by one subject who was highly sensitive to EMS (VAS $=9.5 \mathrm{~cm}$ with EMS vs. $7.1 \mathrm{~cm}$ with ENS). Nonetheless, the reduced discomfort associated with MNS compared to ENS and EMS did not result in differences in MVC force levels when either of these techniques was delivered during MVC. This might appear surprising given the findings reported by Button and Behm (2008), who showed that ENS anticipation resulted in reduced knee extensor MVC force. Our result might be explained by the fact that the present subjects were all very familiar with the testing procedures, as all of them had taken part in at least one previous experiment involving tibial nerve stimulation. In agreement, Button and Behm (2008) reported that VAL and MVC were not significantly different when the superimposed stimulus was anticipated in experienced subjects, contrary to inexperienced subjects. Because discomfort remains an issue especially with sensitive populations, the lower discomfort level induced by MNS is a major advantage when compared to electrical stimulation.
All three stimulation methods have limitations, which must be considered when selecting the appropriate method for in vivo neuromuscular evaluation. For MNS, the nerve trunk must be accessible to the coil, which implies position limitations such as an extended leg for the plantar flexors. In an extended knee position, a different contribution of the triceps surae muscles has been reported in comparison to the knee flexed position (Cresswell et al. 1995). In contrast, ENS and EMS can be used at any joint angle. Another potential concern is the "intra-method" reliability of measurements conducted with these three methods of stimulation; for instance, electrode or coil displacement can lead to increased variability. It is also noteworthy that the present study revealed a high between-subjects variability for some parameters (VAL, $\mathrm{Pt}, \mathrm{PS} 100, \mathrm{PS} 10)$ and this variability associated with a relative small subject sample $(N=10)$ may lead to type II error. Future studies using greater sample sizes and assessing the reproducibility of these methods are thus needed.

In conclusion, MNS and ENS give the same results when assessing neuromuscular function of the plantar flexors in both fresh and fatigued states. In addition, if $100-\mathrm{Hz}$ paired stimuli are used to evaluate contractile properties, MNS, EMS and ENS can be used interchangeably. MNS was found to overcome the major limitation of ENS and EMS, namely self-reported discomfort. The choice between ENS, EMS and MNS for plantar flexor muscle assessment should therefore be based on the study design (e.g. subject position, the use of single or paired stimuli) and on the studied population, MNS being recommended for frail populations.

Acknowledgments The authors would like to thank all the participants who took part in this study and Fabienne Crettaz von Roten for the helpful discussion on the statistical analyses. Daria Neyroud was supported by the Sir Jules Thorn Charitable Trust and John Temesi was supported by a doctoral research grant from the Rhône-Alpes Region.

Conflict of interest The authors declare that they have no conflict of interest.

Ethical standard The study protocol was performed in accordance with the Declaration of Helsinki and approved by the Geneva University Hospital ethics committee (protocol 11-287). Before participation, each subject gave written informed consent. This study complied with the laws of Switzerland.

\section{References}

Allen GM, Gandevia SC, McKenzie DK (1995) Reliability of measurements of muscle strength and voluntary activation using twitch interpolation. Muscle Nerve 18(6):593-600

Andreassen CS, Jakobsen J, Ringgaard S, Ejskjaer N, Andersen H (2009) Accelerated atrophy of lower leg and foot muscles-a follow-up study of long-term diabetic polyneuropathy using magnetic resonance imaging (MRI). Diabetologia 52(6):1182-1191

Bachasson D, Millet GY, Decorte N, Wuyam B, Levy P, Verges S (2013) Quadriceps function assessment using an incremental test 
and magnetic neurostimulation: a reliability study. J Electromyogr Kinesiol 23(3):649-658

Barker AT (1991) An introduction to the basic principles of magnetic nerve stimulation. J Clin Neurophysiol 8(1):26-37

Barker AT, Freeston IL, Jalinous R, Jarratt JA (1987) Magnetic stimulation of the human brain and peripheral nervous system: an introduction and the results of an initial clinical evaluation. Neurosurgery 20(1):100-109

Brouwer B, Wheeldon R, Stradiotto-Parker N, Allum J (1998) Reflex excitability and isometric force production in cerebral palsy: the effect of serial casting. Dev Med Child Neurol 40:168-175

Burke D (2002) Effects of activity on axonal excitability: implications for motor control studies. Adv Exp Med Biol 508:33-37

Button DC, Behm DG (2008) The effect of stimulus anticipation on the interpolated twitch technique. J Sports Sci Med 7:520-524

Cresswell AG, Loscher WN, Thorstensson A (1995) Influence of gastrocnemius muscle length on triceps surae torque development and electromyographic activity in man. Exp Brain Res 105(2):283-290

Elias LJ, Bryden MP, Bulman-Fleming MB (1998) Footedness is a better predictor than is handedness of emotional lateralization. Neuropsychologia 36(1):37-43

Gandevia SC (2001) Spinal and supraspinal factors in human muscle fatigue. Physiol Rev 81(4):1725-1789

Gaudreault N, Gravel D, Nadeau S (2009) Evaluation of plantar flexion contracture contribution during the gait of children with Duchenne muscular dystrophy. J Electromyogr Kinesiol 19(3):e180-e186

Han TR, Shin HI, Kim IS (2006) Magnetic stimulation of the quadriceps femoris muscle: comparison of pain with electrical stimulation. Am J Phys Med Rehabil 85(7):593-599

Harris ML, Luo YM, Watson AC, Rafferty GF, Polkey MI, Green M, Moxham J (2000) Adductor pollicis twitch tension assessed by magnetic stimulation of the ulnar nerve. Am J Respir Crit Care Med 162(1):240-245

Kernell D, Monster AW (1982a) Time course and properties of late adaptation in spinal motoneurones of the cat. Exp Brain Res 46(2):191-196

Kernell D, Monster AW (1982b) Motoneurone properties and motor fatigue. An intracellular study of gastrocnemius motoneurones of the cat. Exp Brain Res 46(2):197-204

Kremenic IJ, Glace BW, Ben-Avi SS, Nicholas SJ, McHugh MP (2009) Central fatigue after cycling evaluated using peripheral magnetic stimulation. Med Sci Sports Exerc 41(7):1461-1466

Laghi F, Harrison MJ, Tobin MJ (1996) Comparison of magnetic and electrical phrenic nerve stimulation in assessment of diaphragmatic contractility. J Appl Physiol 80(5):1731-1742

Man WD, Moxham J, Polkey MI (2004) Magnetic stimulation for the measurement of respiratory and skeletal muscle function. Eur Respir J 24(5):846-860

Martin V, Millet GY, Martin A, Deley G, Lattier G (2004) Assessment of low-frequency fatigue with two methods of electrical stimulation. J Appl Physiol 97(5):1923-1929

Millet GY, Martin V, Martin A, Vergès S (2011) Electrical stimulation for testing neuromuscular function: from sport to pathology. Eur J Appl Physiol 111(10):2489-2500

Millet GY, Bachasson D, Temesi J, Wuyam B, Feasson L, Verges S, Levy P (2012) Potential interests and limits of magnetic and electrical stimulation techniques to assess neuromuscular fatigue. Neuromuscul Disord 22(Suppl 3):S181-S186
Neyroud D, Vallotton A, Millet GY, Kayser B, Place N (2014) The effect of muscle fatigue on stimulus intensity requirements for central and peripheral fatigue quantification. Eur J Appl Physiol 114(1):205-215

Place N, Maffiuletti NA, Martin A, Lepers R (2007) Assessment of the reliability of central and peripheral fatigue after sustained maximal voluntary contraction of the quadriceps muscle. Muscle Nerve 35(4):486-495

Place N, Duclay J, Lepers R, Martin A (2009) Unchanged H-reflex during a sustained isometric submaximal plantar flexion performed with an EMG biofeedback. J Electromyogr Kinesiol 19(6):395-402

Place N, Casartelli N, Glatthorn JF, Maffiuletti NA (2010) Comparison of quadriceps inactivation between nerve and muscle stimulation. Muscle Nerve 42(6):894-900

Polkey MI, Kyroussis D, Hamnegard CH, Mills GH, Green M, Moxham J (1996) Quadriceps strength and fatigue assessed by magnetic stimulation of the femoral nerve in man. Muscle Nerve 19(5):549-555

Polkey MI, Duguet A, Luo Y, Hughes PD, Hart N, Hamnegard CH, Green M, Similowski T, Moxham J (2000) Anterior magnetic phrenic nerve stimulation: laboratory and clinical evaluation. Intensive Care Med 26(8):1065-1075

Rodriguez-Falces J, Maffiuletti NA, Place N (2013a) Spatial distribution of motor units recruited during electrical stimulation of the quadriceps muscle versus the femoral nerve. Muscle Nerve 48(5):752-761

Rodriguez-Falces J, Maffiuletti NA, Place N (2013b) Twitch and $\mathrm{M}$-wave potentiation induced by intermittent maximal voluntary quadriceps contractions: differences between direct quadriceps and femoral nerve stimulation. Muscle Nerve 45:752-761

Rutherford OM, Jones DA, Newham DJ (1986) Clinical and experimental application of the percutaneous twitch superimposition technique for the study of human muscle activation. J Neurol Neurosurg Psychiatry 49(11):1288-1291

Scaglioni G, Martin A (2009) Assessment of plantar flexors activation capacity: nerve versus muscle stimulation by single versus double pulse. Eur J Appl Physiol 106(4):563-572

Strojnik V, Komi PV (1998) Neuromuscular fatigue after maximal stretch-shortening cycle exercise. J Appl Physiol 84(1):344-350

Swallow EB, Gosker HR, Ward KA, Moore AJ, Dayer MJ, Hopkinson NS, Schols AM, Moxham J, Polkey MI (2007) A novel technique for non volitional assessment of quadriceps muscle endurance in humans. J Appl Physiol 103(3):739-746

Tomazin K, Verges S, Decorte N, Oulerich A, Maffiuletti NA, Millet GY (2011) Fat tissue alters quadriceps response to femoral nerve magnetic stimulation. Clin Neurophysiol 122(4):842-847

Vagg R, Mogyoros I, Kiernan MC, Burke D (1998) Activity-dependent hyperpolarization of human motor axons produced by natural activity. J Physiol 507:919-925

Verges S, Maffiuletti NA, Kerherve H, Decorte N, Wuyam B, Millet GY (2009) Comparison of electrical and magnetic stimulations to assess quadriceps muscle function. J Appl Physiol 106(2):701-710

Vinci P, Perelli SL (2002) Footdrop, foot rotation, and plantar flexor failure in Charcot-Marie-Tooth disease. Arch Phys Med Rehabil 83(4):513-516

Wiley ME, Damiano DL (1998) Lower-extremity strength profiles in spastic cerebral palsy. Dev Med Child Neurol 40(2):100-107 